\title{
Cell patterning via laser micro/nano structured silicon surfaces
}

\author{
Ch. Yiannakou ${ }^{1,2}$, Ch. Simitzi ${ }^{1}$, A. Manousaki ${ }^{1}$, C. Fotakis ${ }^{1,2}$, A. Ranella ${ }^{1}$ and E. Stratakis ${ }^{1,3^{*}}$ \\ ${ }^{1}$ Institute of Electronic Structure and Laser, Foundation for Research and Technology - Hel- \\ las, Heraklion, 71110, Crete, Greece \\ ${ }^{2}$ Department of Physics, University of Crete, Heraklion, 71003, Crete, Greece \\ ${ }^{3}$ Department of Materials Science and Technology, University of Crete, Heraklion, 71003 \\ Crete, Greece
}

\begin{abstract}
The surface topography of biomaterials can have an important impact on the cellular adhesion, growth and proliferation. Apart from the overall roughness, the detailed morphological features at all length scales significantly affect the cell-biomaterial interactions in a plethora of applications including structural implants, tissue engineering scaffolds and biosensors. In this study, we present a simple, one-step direct laser patterning technique to fabricate nanoripples and dual-rough hierarchical micro/nano structures to control SW10 cell attachment and migration. It is shown that, depending on the laser processing conditions, distinct cell-philic or cell-repellant patterned areas can be attained with a desired motif. We envisage that our technique could enable spatial patterning of cells in a controllable manner, giving rise to advanced capabilities in cell biology research.
\end{abstract}

\section{Introduction}

The in vivo extracellular environment features both biochemical and physical cues to the cells, including topographical and mechanical ones. In principle, such cues can be identified since cells respond to them by initiating a sequence of different processes including change of cellular functions and shape. The effect of a topographical cue, as this can be dictated by a geometrical discontinuity or anisotropy, on the cellular shape has been known since almost 100 years and the phenomenon is termed as contact guidance [1-4].

Currently, the emergence of micro- and nano-fabrication techniques enables the precise surface patterning at the cellular and the subcellular scale. For this purpose, various approaches have been implemented, using variant materials like polymers, ceramics, or metals and fabrication techniques including photolithography, electrospinning and laser 
structuring [5-8]. As a result, patterning of different topographical features such as grooves, pillars, cones and channels, as well as micro/nano geometries, have been realized onto materials' surfaces. Such textured surfaces have been widely used as cell culture platforms for the in vitro study of cellular responses on topographical cues [8-12]. Based on increasing number studies, surface topography has been shown to affect the cellular morphology, orientation and in turn cellular functions, such as adhesion, proliferation, migration and differentiation [9,11,1,13-16]. Furthermore, cells become polarized along continuous anisotropic topographical features, in the form of parallel polymeric fibers or grooves, or discontinuous anisotropic features in the form of elliptical cones [17]. In our previous work, we have studied the effect of surface topographical patterns on the morphology, outgrowth and differentiation of nerve and neuroglial cells $[15,17,18]$. It was found that a discontinuous topographical pattern could promote axonal alignment as well as influence the cell differentiation process.

A less investigated topographical model is the so-called hierarchical or multiscale one, which is described by a topography comprising morphological features at various lengthscales. Although difficult to study, hierarchical morphologies can better recapitulate the complex topography of the extracellular matrix [19]. Indeed, hierarchical topographies on different materials comprising microsized and nanosized features have been shown to influence the elongation, proliferation and/or differentiation of endothelial cells [20] osteoblasts [21], neural phenotype cells and stem cells [22-24]. In these studies, hierarchical patterns have been developed via multiple step processes, based on the growth of nanotopographical features on initially fabricated micropatterns or via chemical modification routes.

At the same time, cell patterning at the intercellular to tissue level has currently been a challenge for tissue engineering applications [25]. Indeed, the ability to control the spatial arrangement of different cell types, while keeping them separated and in close proximity for the culture time, paves the way for the development of patterned co-cultures [26]. Such culture systems enable the precise control of homotypic and heterotypic contact and the study of cell-cell interactions in vitro and thus can better mimic the complexity of the in vivo cellular microenvironment [26]. Various approaches of selective cell adhesion guided by topographical [11,27] and/or (bio)chemical [28,29] cues have been studied.

In this study we first demonstrate a simple, one-step method of fabricating hierarchical structures, based on ultrafast laser structuring of silicon in water. Laser processing in liquid environments is a straightforward room temperature technique. This attribute makes it attractive to generate a variety of surface morphologies practically on any material [30]. 
Specifically, we show that by varying the laser processing fluence, different textured surfaces can be realized, ranging from nanorippled (at low fluences) to hierarchical patterns (at high fluences) comprising micro-grooves decorated with nano-ripples. The fabricated surfaces have been used as substrates for culturing neuroglial SW10 cells. It is shown that, although cell adhesion and growth is inhibited by the nano-sized features, it is significantly promoted by the hierarchical micro- and nano- scaled structures. More importantly, this surface topography-induced control over selective cell adhesion enables the realization of arbitrary cell patterns, on demand, onto a single culture substrate, as a first step for the development of patterned co-culture platforms via computer-aided scanning of the laser beam.

\section{Materials and Methods}

\subsection{Fabrication and characterization of micro-nano structured Si surfaces}

Single crystal n-type Si (100) wafers of $500 \mu \mathrm{m}$ thickness were used for the laser irradiation experiments. The wafers were placed inside a glass Petri dish filled with deionized Millipore water up to a $4 \mathrm{~mm}$ height. The irradiating laser source was constituted by a regenerative amplified Ti:Sapphire $(\lambda=800 \mathrm{~nm})$, delivering $150 \mathrm{fs}$ pulses at a repetition rate of $1 \mathrm{kHz}$. The processing chamber was placed on a computer-driven high precision X-Y translation stage (Standa) with spatial resolution of $1 \mu \mathrm{m}$ allowing sample displacement with regard to the laser beam up to $100 \mathrm{~mm}$. The laser fluence used in these experiments was in the range of 0.20 to $0.97 \mathrm{~J} / \mathrm{cm}^{2}$.

In order to evaluate cell response on different surfaces under identical cell culture conditions, each laser-treated Si surface contained four bands of $3 \times 3 \mathrm{~mm}^{2}$, fabricated at four different laser fluence values. Flat non-patterned silicon has been used as control.

Following laser irradiation, the laser-patterned surfaces were morphologically characterized by field emission scanning electron microscopy (SEM) (JEOL 7000F). An image-processing algorithm (ImageJ, National Institutes of Health, Bethesda, MD, USA) was implemented to determine the morphological characteristics of the structured surfaces, including periodicity $(\Lambda)$ and height $(\mathrm{h})$ from top, side-view and cross-sectional SEM images. The respective contact angles $(\theta)$ were calculated via an automated tension meter using the sessile droplet method. Specifically, a $2 \mu$ distilled, deionized Millipore water droplet was gently positioned on the surface, using a microsyringe, and images were captured to measure the angle formed at the liquid-solid interface. The mean value was 
calculated from at least five individual measurements. Successive measurements were reproducible within $\pm 1^{\circ}$.

Table 1: Geometrical characteristics and wettability of the different laser-patterned substrates

\begin{tabular}{cccc}
\hline \multirow{2}{*}{ Type of roughness } & \multicolumn{2}{c}{ Geometrical Characteristics } & Wetting Angle \\
& Periodicity & Height & Contact angle \\
& $\boldsymbol{\Lambda} \pm \mathbf{S D}(\mathbf{n m})$ & $\boldsymbol{h} \pm \mathbf{S D}(\boldsymbol{\mu m})$ & $\boldsymbol{\theta} \pm \mathbf{S D}\left({ }^{\mathbf{o}}\right)$ \\
\hline Ripples & $146 \pm 37$ & - & $47 \pm 1$ \\
Low micro-Grooves & $152 \pm 34$ & $2.4 \pm 1.1$ & $43 \pm 1$ \\
Medium micro-Grooves & $142 \pm 42$ & $5.5 \pm 0.6$ & $43 \pm 1$ \\
High micro-Grooves & $146 \pm 46$ & $11.0 \pm 1.5$ & $49 \pm 1$ \\
\hline
\end{tabular}

\subsection{Cell culture}

Schwann cells (SW10) were maintained in Dulbecco's modified Eagle's medium (DMEM; Invitrogen, Grand Island, NY, USA) supplemented with $10 \%$ fetal bovine serum (FBS; Biosera, Sussex, UK) at $33^{\circ} \mathrm{C}$ in a $5 \% \mathrm{CO}_{2}$ atmosphere. In order to minimize interassay variability, low passage number (P 5-7) cells were used throughout the study. Structured surfaces were sterilized in an autoclave and transferred into sterile culture wells 48 well plates (Sarstedt; Numbrecht, Germany). SW10 cells were seeded onto the silicon substrates at a density of $5 \times 10^{4}$ cells $/ \mathrm{ml}$ and were cultured for 4 days. No protein coating has been used.

\subsection{Immunocytochemical assays and cell counting}

SW10 cells were double-stained for F-actin and S100. After 3 days of culture the cells were fixed with $4 \%$ PFA for $15 \mathrm{~min}$ and permeabilized with $0.1 \%$ Triton X-100 in PBS for 3-5 min. The non-specific binding sites were blocked with 2\% BSA in PBS for $30 \mathrm{~min}$. The S100 was detected by incubating the cells with the anti-S100b (1:500 Abcam, Rab Mab ab52642) for $1 \mathrm{~h}$ at RT. After 2 washes with PBS, specimens were incubated for $1 \mathrm{~h}$ at RT with Alexa Fluor 546 goat anti-rabbit IgG (MoBiTec A-11010) for $45 \mathrm{~min}$ at RT. Simultaneously, the cells were incubated with tetramethyl rhodamine isothiocyanateconjugated phalloidin (1:400 in PBS-BSA 2\%; Biotium) for F-actin staining. The samples were then washed with PBS and mounted on coverslips with antifade reagent containing DAPI for nuclei staining (ProLong® Gold reagent; Molecular Probes, Eugene, OR, USA). Cell imaging was performed using an epifluorescence microscope coupled to a high-resolution Carl Zeiss Axiocam colour camera; 10, 20 and 40x objectives were used. 


\subsection{Scanning electron microscopy of cells}

Following culture termination, the cells were washed with $0.1 \mathrm{M}$ sodium cacodylate buffer (SCB) and then incubated in the same solution for $15 \mathrm{~min}$. After repeating this step twice, the cells were fixed using $2 \%$ glutaraldehyde, $2 \%$ formaldehyde in $1 \%$ SCB fixative buffer for $1 \mathrm{~h}$ at $4^{\circ} \mathrm{C}$. All surfaces were then washed twice (for 15 min each time) with $1 \%$ SCB at $4{ }^{\circ} \mathrm{C}$, dehydrated by immersion in serially graded ethanol solutions (50-100\%) and incubated for $15 \mathrm{~min}$ in dry $100 \%$ ethanol. Prior to SEM examination, the samples were sputter-coated with a $10 \mathrm{~nm}$ gold layer.

\subsection{Image processing and Statistical analysis}

Quantitative information regarding cell number was assessed using an imageprocessing algorithm (ImageJ v1.36). Specifically, the number of nuclei per surface area was calculated using the "Cell Counter" plugin. The results represent the means of at least three different experiments ( $\mathrm{n}=\sim 30$ fields of view for each substrate type). For statistical analysis, the data were subjected to one-way ANOVA followed by Tukey tests for multiple comparisons between pairs of means, using commercially available software (SPSS 21, IBM). Statistically significant difference between experimental results was indicated by $\mathrm{p}<$ 0.05 . The results are expressed as means \pm standard error (SE).

\section{Results and discussion}

Direct laser processing via ultra-short laser pulses can give rise to a plethora of patterns on the surfaces of various semiconducting and metallic materials, via simply tuning the laser parameters and irradiation environment [30]. It is a fabrication approach with high reproducibility and precision, due to the absence of heat exchange and thermal effects which would cause collateral damage to the surrounding area [31]. Furthermore, the patterns can be easily transferred to various types of soft polymeric materials via soft lithography approaches [12].

Femtosecond laser irradiation of a $\mathrm{Si}$ wafer immersed in water leads to surfaces exhibiting various morphological and wetting characteristics (Table 1). In particular, at low laser fluences the resulting surfaces consist of periodic nano-sized ripples, exhibiting a period that is much lower than the laser wavelength [32]. However, as the laser fluence increases quasi-periodical micro-grooves with increasing height were formed [33]. 


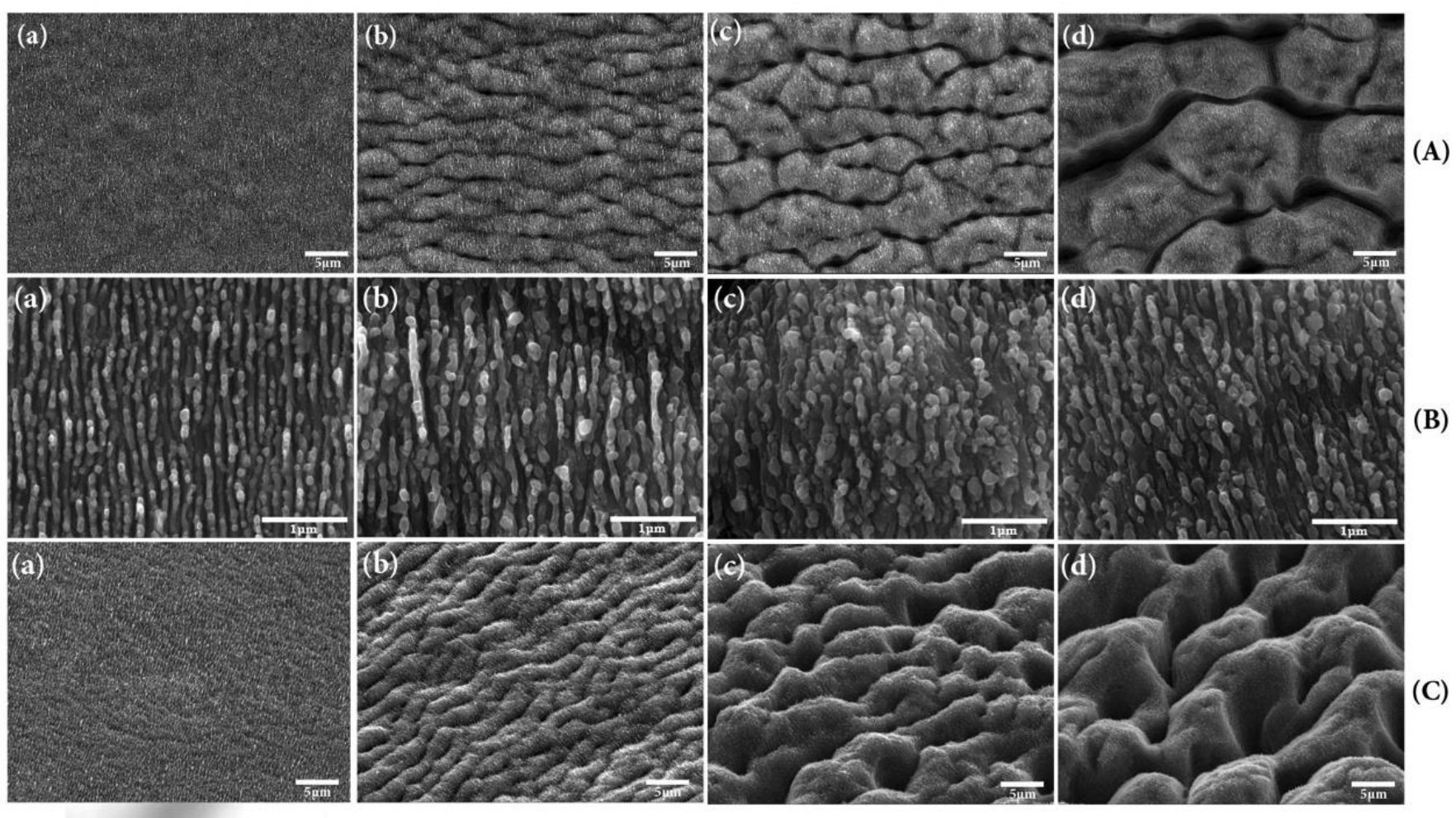

Figure 1: SEM micrographs of the various laser-patterned Si surfaces fabricated at $0.20 \pm 0.10 \mathrm{~J} / \mathrm{cm}^{2}$, denoted as NR areas (a), $0.55 \pm 0.10 \mathrm{~J} / \mathrm{cm}^{2}$, denoted as LM areas (b), $0.62 \pm 0.14 \mathrm{~J} / \mathrm{cm}^{2}$, denoted as MM areas (c) and $0.88 \pm 0.24 \mathrm{~J} / \mathrm{cm}^{2}$, denoted as HM areas (d). (A) and (B) represent low- and highmagnification top views, while $(\mathrm{C})$ shows the respective side $\left(45^{\circ}\right)$ views.

In this work low- (LM), medium- (MM) and high- (HM) height micro-grooved surfaces have been studied (Figure 1). As calculated from SEM images, micro-grooved height varied from $2.42 \pm 1.07 \mu \mathrm{m}$ for the LM to $10.98 \pm 1.52 \mu \mathrm{m}$ for the HM structures (Table 1). Remarkably, the micro-grooved structures were decorated with nanoripples of similar periodicity, resulting into hierarchically micro/nano-patterned surfaces (Figure 1B). Thus, by changing only a single irradiation parameter, i.e. laser fluence, the different patterns were directly designed on the silicon surface.

Static contact angle measurements showed that all fabricated surfaces were quite hydrophilic exhibiting similar wettability (Table1). This indicates that the surface chemistry is similar among the different patterned surfaces. The observed hydrophilicity can be attributed to a layer of natural oxide. This layer is formed on surfaces being irradiated by short laser pulses in water environment and its thickness is of the order of a few nanometers. [30,34]. This oxide layer protects them from chemical interaction with both water and air oxygen. When irradiating in water environment, water layer height is critical because it impacts the laser ablation rate [35]. In the present study water height has been kept constant at $4 \mathrm{~mm}$. 
SW10, a mouse neuronal Schwann cell line was employed to assess cellular growth on the laser-patterned substrates. Cell growth was evaluated both qualitatively, via immunostaining for the protein S100b and the actin and via SEM imaging, as well as quantitatively, via image analysis of DAPI nuclear staining.

(A)
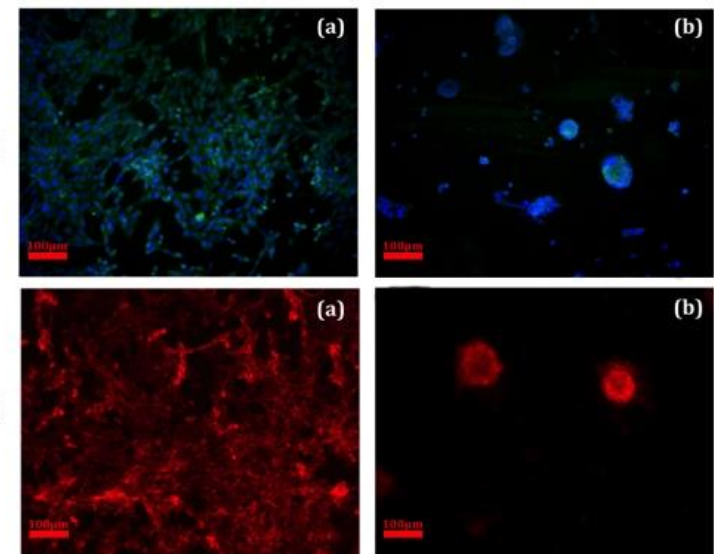

(a)

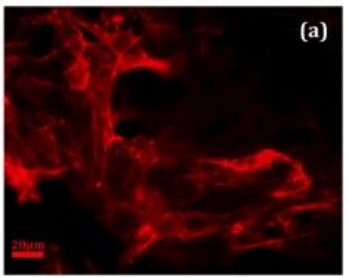

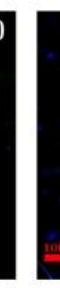
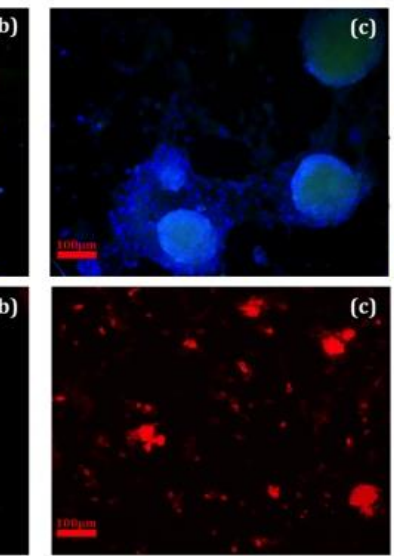

(b)

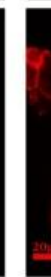

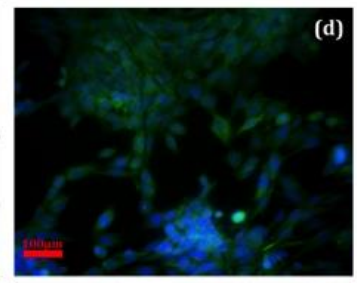
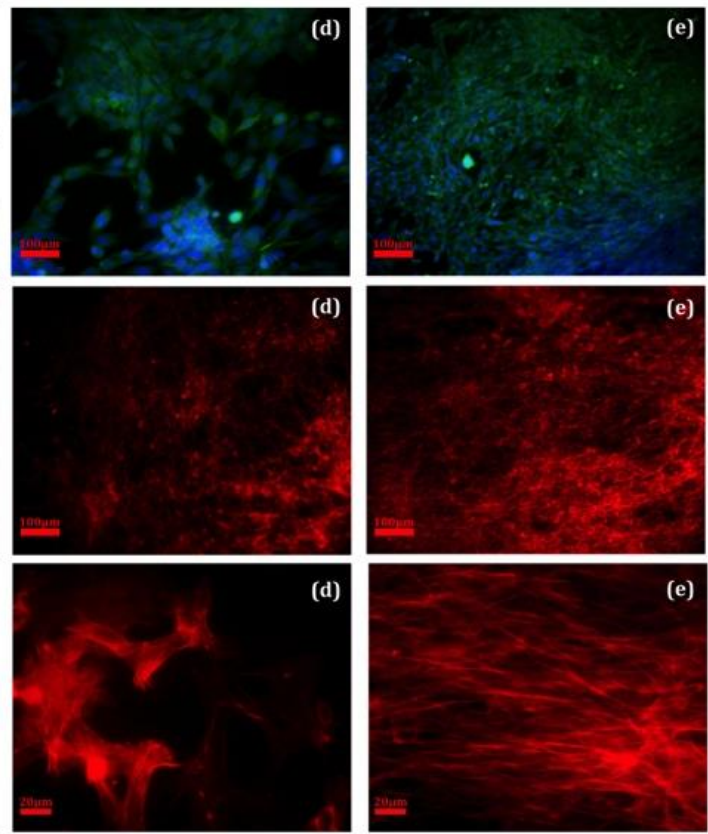

Figure 2: A) Fluorescence microscopy images of SW10 cells cultured on flat (a), NR- (b), LM- (c) MM- (d), HM- (e) patterned Si substrates fabricated at different laser fluences. The cells were double labelled for S100 (green) and DAPI (blue) antibodies; (B) Respective fluorescence microscopy images of cells labelled for actin (red); (C) High magnification images of (B).

As shown in Figure 2, there was a differential cell response and outgrowth on the various substrates. More specifically, cells adhered and grew well onto the flat unpatterned Si substrates, however, without extended spreading (Figure $2 \mathrm{Ba} \& 2 \mathrm{Ca}$ ). On the contrary, cell outgrowth was inhibited on the laser-patterned nanorippled substrates; the cells remained round-shaped and were assembled into small clusters sparsely distributed on the surface (Figure $2 \mathrm{Bb} \& 2 \mathrm{Cb}$ ). With increasing laser fluence, which results in combined micro(grooves) and nano- (ripples) roughness, cell outgrowth was significantly improved. In this case, cells developed numerous extensions, exhibiting morphological characteristics comparable with those of the cells on the unpatterned control surfaces (Figure 2Bc,d 2Cc,d). Given that the microgrooves are also terminated with nanoripples exhibiting similar periodicity to that of the nanorippled substrates, the above results demonstrate the significant role of hierarchical morphology, i.e. the combination of micro- and nano- features, on cell outgrowth. 

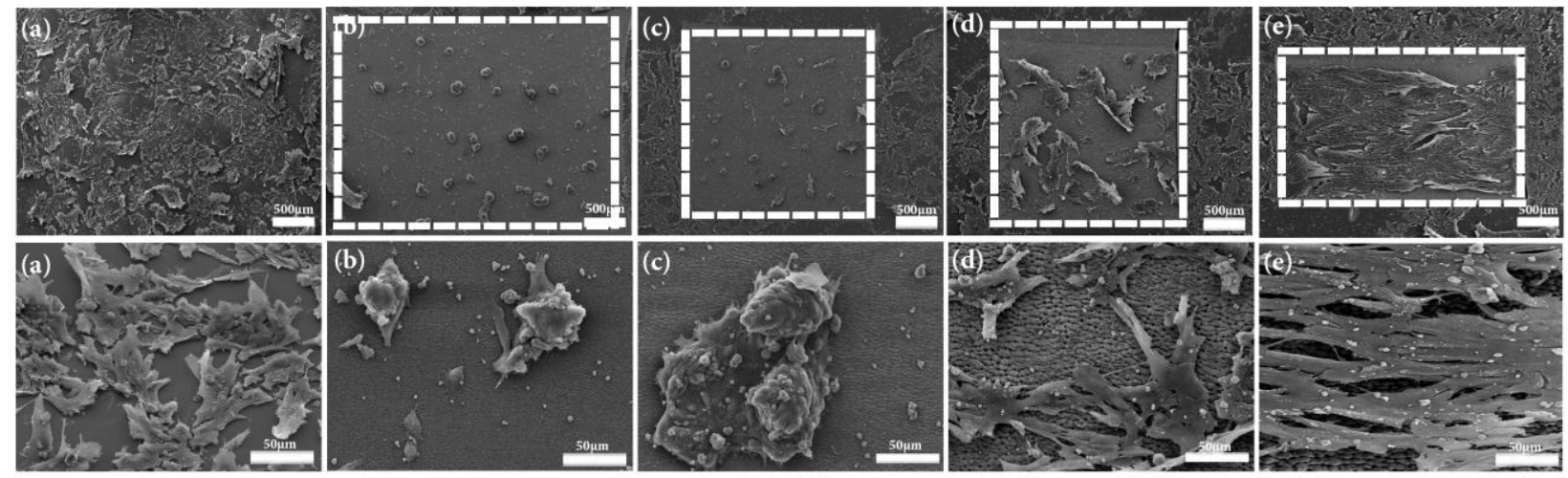

(A
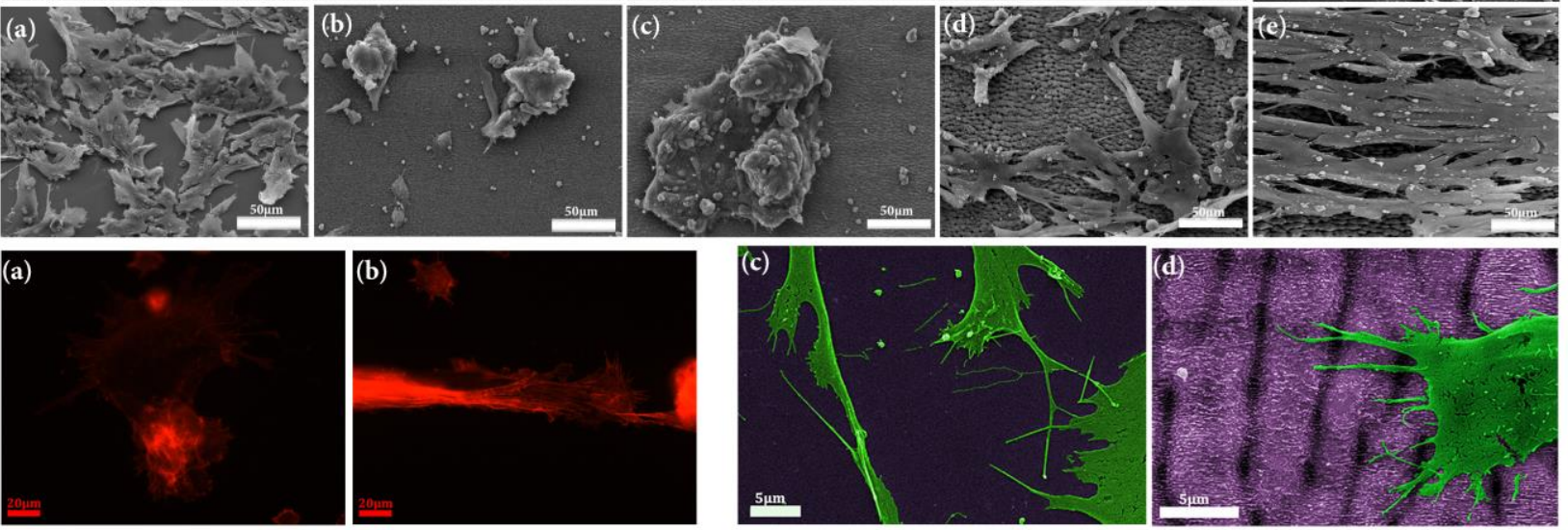

Figure 3: $(\mathrm{A}, \mathrm{B})$ Top view SEM images of $\mathrm{SW} 10$ cells on the flat $(\mathrm{A}, \mathrm{Ba})$ and the $\mathrm{Si}$ patterned surfaces NR (A,Bb), LM (A,Bc), MM (A,Bd) and HM (A,Be). (A) and (B) are low and highmagnification images, respectively. The laser treated areas are marked with the dashed rectangles. (C) Fluorescence microscopy images of actin-labeled SW10 cells on flat $\mathrm{Si}(\mathrm{Ca})$ and on $\mathrm{HM}$ roughness $(\mathrm{Cb})$; Pseudo-colored top-view SEM images of SW10 cells on flat Si $(\mathrm{Cc})$ and HM substrate $(\mathrm{Cd})$.

Among the various patterned substrates the HM were the most favourable substrate for the cell outgrowth in terms of cell number and morphology. Cells on the HM substrates completely covered the micropatterned area (Figure $2 \mathrm{Be} \& 2 \mathrm{Ce}$ ). This topographydependent cell response was further confirmed by SEM imaging (Figure 3). Additionally, as shown in the high magnification SEM micrographs of Figure $3 \mathrm{Cc}$ and $3 \mathrm{Cd}$, cells on both surfaces exhibit dense filopodia extensions.

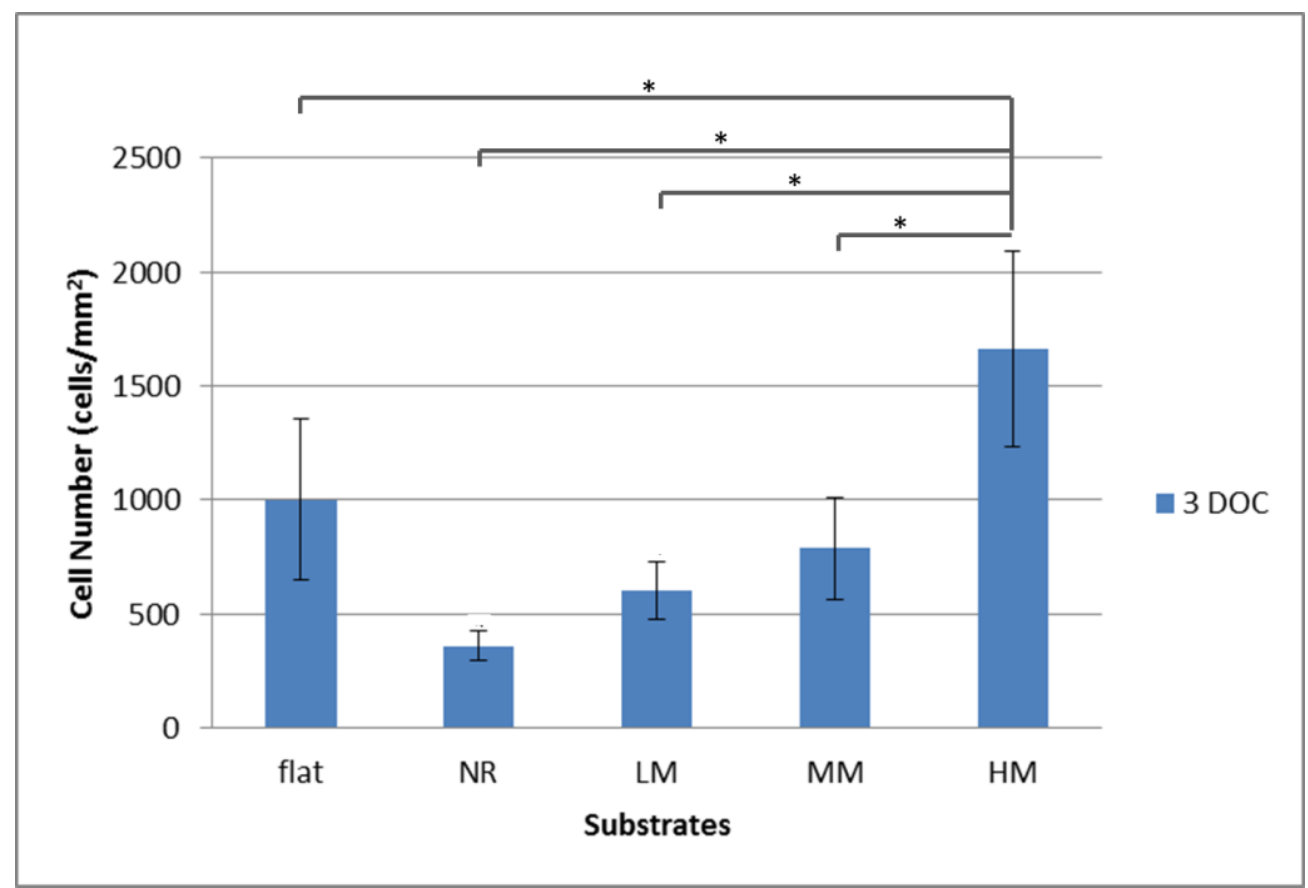


Figure 4: Number of SW10 cells growing on the laser-patterned Si substrates for 3 days of culture (DOC). Upon culture termination, the scaffolds were stained with DAPI and the number of nuclei per surface area was evaluated using the ImageJ analysis software. The results are expressed as cell numbers $/ \mathrm{mm}^{2}$ ( \pm standard error of the mean, SE and represent the means of five different experiments $(n=30$ fields of view for each substrate type). The difference between HM and all the surfaces was significant $(*: \mathrm{p}<0.05)$. The difference between flat surface and NR, LM and HM surfaces was significant $*: \mathrm{p}<0.05)$.

In order to quantitatively evaluate cell outgrowth on the different substrates, upon culture termination, scaffolds were stained with DAPI and the number of nuclei per $\mathrm{mm}^{2}$ of surface area was evaluated using ImageJ analysis. The results are presented in Figure 4, showing the mean cell number in each case tested, i.e. flat, NR, LM, MM, HM. It is clear that cell outgrowth was significantly improved on the HM patterned areas, contrary to the nano-rippled ones, which are cell-repellent. Thus, together these results demonstrate the significant role of the hierarchical morphology, i.e. the combination of micro- and nanofeatures, on favoring cell outgrowth.

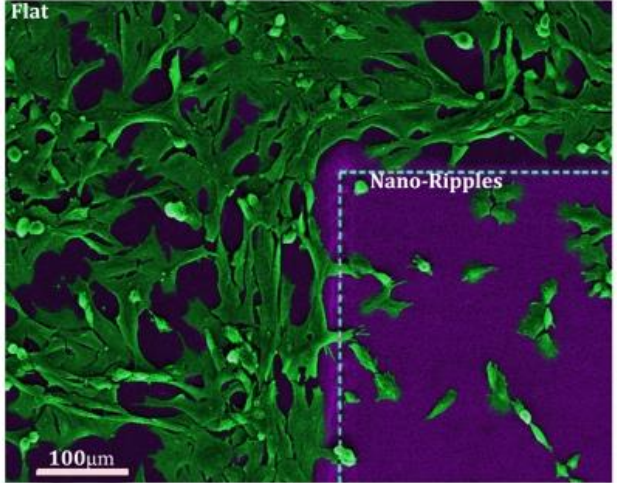

(A)

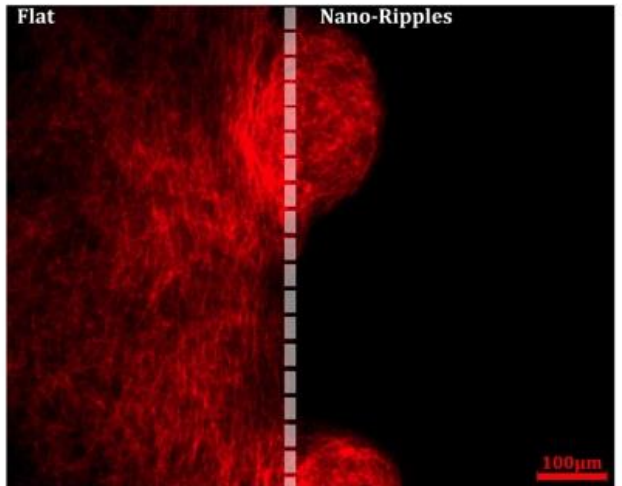

(B)

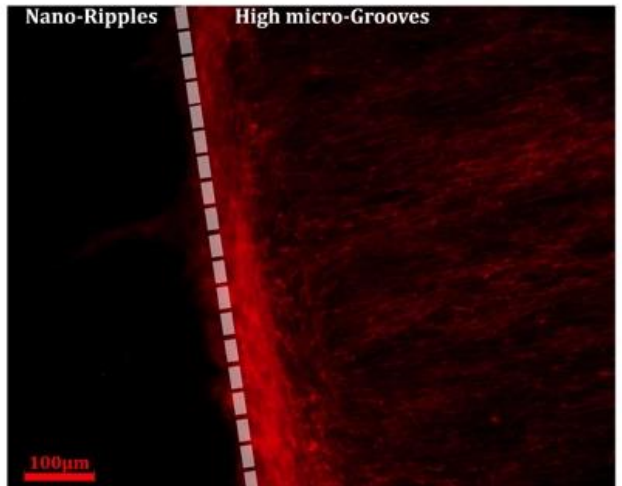

(C)

Figure 5: (A) Pseudo-colored top view SEM images SW10 cells grown onto a square-shaped area of NR, fabricated on flat Si; (B) Fluorescence microscopy image of actin-labelled SW10 cells grown onto a non-adherent NR area, fabricated on adherent flat Si; (C) Fluorescence microscopy image of actin-labelled SW10 cells grown onto a laser patterned Si substrate consisting of non-adherent NR and adherent HM patterned areas.

Hierarchical topographies attain increasing interest in the design of cell culture platforms and/or implants, since they can better mimic the structural complexity of tissues [19]. The majority of the studies include surface texturing of titanium surfaces to study/understand the cell-biomaterials interactions for improving the performance of the implants in the field of joint prostheses or facture fixation devices [36-38]. Accordingly, titanium disks consisting of micropits with added nanonodules, ranging from 100 to $500 \mathrm{~nm}$, enhanced osteoblast but not fibroblast function compared to the single micropits [38]. Furthermore, hierarchical Ti-based substrates comprising pits decorated with nanostructures, which have been fabricated by sandblasting and etching, improved the initial adhesion and 
proliferation of pre-osteoblasts and their late osteogenic differentiation compared to the single micropits [37]. Beyond bone tissue, the effect of hierarchical topography on cell functions compared to the single micro-or nano-scale patterns is increasingly studied in various cell types and material systems. Methacrylate-based polymeric/ HEMA-EDMA polymeric patterns consisting of large agglomerates decorated with porous sponge-like nanostructures helped the embryonic stem cells to retain their pluripotency state, while the
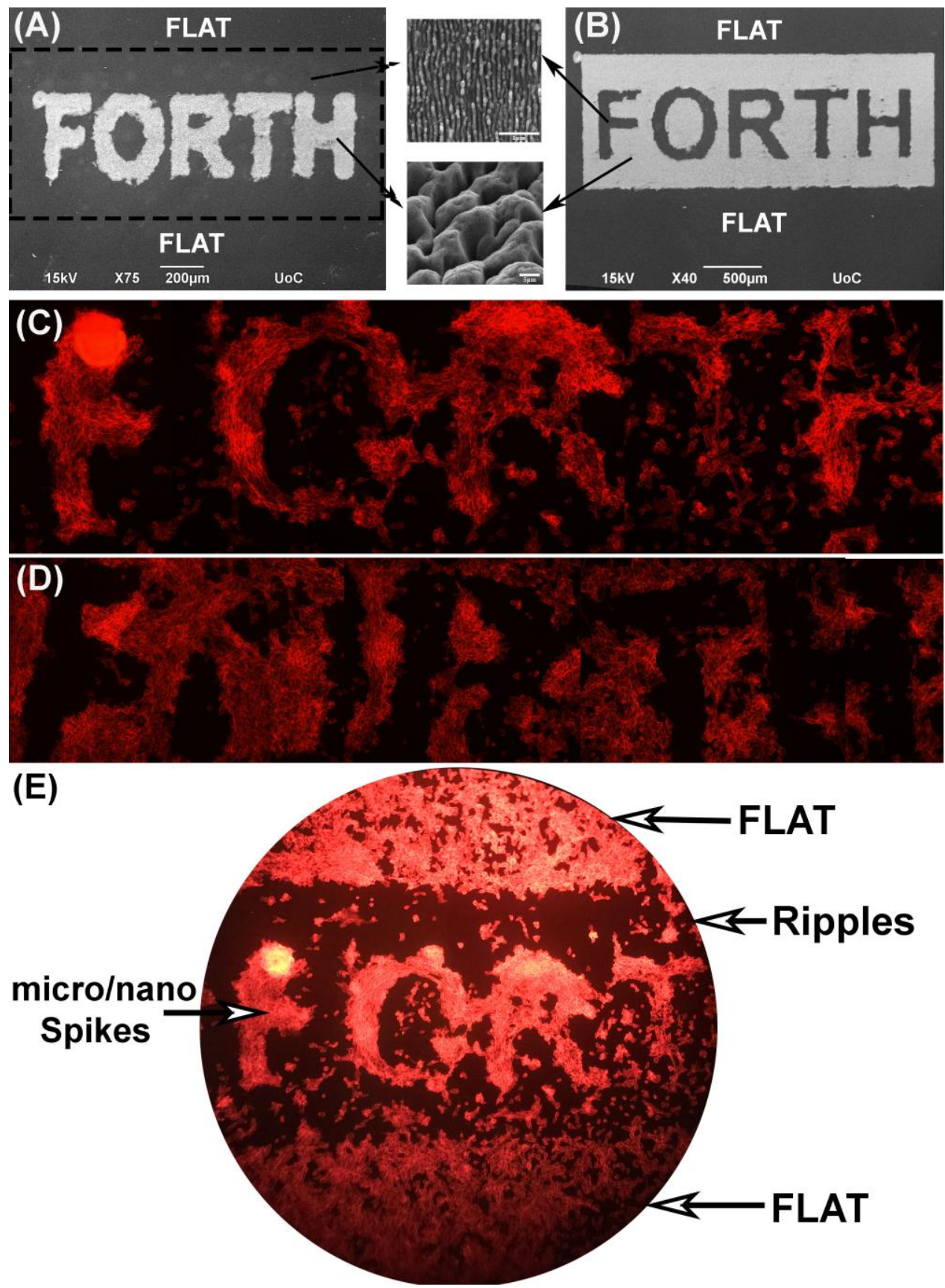

Figure 6: (A) Top view SEM image of a custom-shaped area comprising HM-structured areas shaping the FORTH logo surrounded by a rectangular-shaped NR area; the flat areas are also indicated; (B) The negative shape of (A), i.e NR-structured areas shaping the FORTH logo, surrounded by a rectangular-shaped HM area; (C and E) Fluorescence microscopy image of actinlabelled SW10 cells grown onto the substrate shown in (A); (D) Fluorescence microscopy image of actin-labelled SW10 cells grown onto the substrate shown in (B). 
smooth or only the nano-patterned surfaces led to fast differentiation of cells [23].

The remarkable dependence of cell outgrowth and substrate roughness at both the micro- and the nano- scales as presented in our work enables the realization of arbitrary cell patterns onto a single culture substrate. This can be simply realized via scanning the laser beam onto the substrate surface and writing in a single-step the desired pattern which comprises interfaces of NR (cell-repellent) with flat and/or HM regions (cell-philic). Examples of cell patterns attained using this process are presented in the scanning electron and fluorescence microscopy images of Figure 5. For instance, in Figures 5A and B, cells tend to avoid the NR-textured area and grow solely on the neighboring flat region. The differential cell responses on the various topographical patterns can be realized via the fabrication of proper interfaces between NR and HM patterned regions (Figure 5C), proving the selectivity of the fabrication technique. More importantly, as presented in Figure 6, arbitrary shaped cell patterns can be realized depending on the structuring motif. Indeed, in Figure 6C, cells migrated to the cell philic area and formed the 'FORTH' logo, while in Figure 6D the negative motif of that shown in Figure 6C was shaped by the cultured cells.

Such sharp cell-philic to cell-repellant interfaces could pave the way for the design of patterned co-cultures based on surface topographical cues. Various engineering approaches are implemented to control the spatial arrangement of cells in order to generate patterned cocultures (reviewed in [26]). Most of such approaches are mainly based on the differential preference of the various cells to specific ECM molecules. In this work, cell patterning is guided by only surface topographical cues and without any chemical treatment. Although we report on the SW10 selective cell adhesion, the use of the laser-patterned substrates on other cell types is highly anticipated. This could bring about new knowledge on the underlying cellular adhesion mechanism.

Our results suggest that laser patterns can be effectively designed to generate separate cell repellant and cell-philic areas and induce guided cell migration into a desired shape or area.

\section{Conclusions}

A novel direct laser writing technique to realise spatial patterns of cells with a desired motif onto a single culture substrate, has been demonstrated. It is based on the controllable fabrication of cell-repellent nanoripples areas combined with cell-philic hierarchical micro/nano structures. The simplicity of our technique makes it potentially attractive 
towards controlling cell patterning with desired shapes or patterns as well as understanding fundamental mechanisms behind cell adhesion, sensing and migration.

\section{Acknowledgements}

This work was supported by the European Research Infrastructure NFFA-Europe, funded by EU's H2020 framework programme for research and innovation under grant agreement $n$. 654360 .

\section{References}

[1] Curtis A and Wilkinson C 1997 Topographical control of cells Biomaterials 18 157383

[2] Harrison R G 1914 The reaction of embryonic cells to solid structures J. Exp. Zool. $17521-44$

[3] Weiss P 1945 Experiments on cell and axon orientation in vitro: The role of colloidal exudates in tissue organization J. Exp. Zool. $100353-86$

[4] Simitzi C, Ranella A and Stratakis E 2017 Controlling the morphology and outgrowth of nerve and neuroglial cells : The effect of surface topography Acta Biomater.

[5] Stratakis E, Ranella A and Fotakis C 2011 Biomimetic micro/nanostructured functional surfaces for microfluidic and tissue engineering applications

Biomicrofluidics 5 1-31

[6] Ross A M, Jiang Z, Bastmeyer M and Lahann J 2012 Physical aspects of cell culture substrates: Topography, roughness, and elasticity Small 8 336-55

[7] Norman J J and Desai T A 2006 Methods for fabrication of nanoscale topography for tissue engineering scaffolds Ann. Biomed. Eng. 34 89-101

[8] Nikkhah M, Edalat F, Manoucheri S and Khademhosseini A 2012 Engineering microscale topographies to control the cell-substrate interface Biomaterials $\mathbf{3 3} 5230$ 46

[9] Ranella A, Barberoglou M, Bakogianni S, Fotakis C and Stratakis E 2010 Tuning cell adhesion by controlling the roughness and wettability of 3D micro/nano silicon structures Acta Biomater. 6 2711-20

[10] Park T H and Shuler M L 2003 Integration of cell culture and microfabrication technology Biotechnol Prog 19 243-53

[11] Jeon H, Koo S, Reese W M, Loskill P, Grigoropoulos C P and Healy K E 2015 Directing cell migration and organization via nanocrater-patterned cell-repellent 
interfaces. Nat. Mater. 14 918-23

[12] Koufaki N, Ranella A, Aifantis K E, Barberoglou M, Psycharakis S, Fotakis C and Stratakis E 2011 Controlling cell adhesion via replication of laser micro/nanotextured surfaces on polymers Biofabrication 345004

[13] Kaiser J P, Reinmann A and Bruinink A 2006 The effect of topographic characteristics on cell migration velocity EMPA Act. 2741

[14] Metavarayuth K, Sitasuwan P, Zhao X, Lin Y and Wang Q 2016 Influence of Surface Topographical Cues on the Differentiation of Mesenchymal Stem Cells in Vitro. ACS Biomater. Sci. Eng. 2 142-51

[15] Simitzi C, Stratakis E, Fotakis C, Athanassakis I and Ranella A 2015 Microconical silicon structures influence NGF-induced PC12 cell morphology J. Tissue Eng. Regen. Med. 9 424-34

[16] Ventre M, Natale C F, Rianna C and Netti P A 2014 Topographic cell instructive patterns to control cell adhesion, polarization and migration J. R. Soc. Interface 11 20140687

[17] Simitzi C, Efstathopoulos P, Kourgiantaki A, Ranella A, Charalampopoulos I, Fotakis C, Athanassakis I, Stratakis E and Gravanis A 2015 Laser fabricated discontinuous anisotropic microconical substrates as a new model scaffold to control the directionality of neuronal network outgrowth Biomaterials 67 115-28

[18] Papadopoulou E L, Samara A, Barberoglou M, Manousaki A, Pagakis S N, Anastasiadou E, Fotakis C and Stratakis E 2010 Silicon Scaffolds Promoting ThreeDimensional Neuronal Web of Cytoplasmic Processes Tissue Eng. Part C-Methods 16 497-502

[19] Wang L and Carrier R L 2011 Biomimetic Topography : Bioinspired Cell Culture Substrates and Scaffolds Advances in Biomimetics pp 453-72

[20] Moffa M, Sciancalepore A G, Passione L G and Pisignano D 2014 Combined nanoand micro-scale topographic cues for engineered vascular constructs by electrospinning and imprinted micro-patterns Small 10 2439-50

[21] Jeon H J, Simon C G and Kim G H 2014 A mini-review: Cell response to microscale, nanoscale, and hierarchical patterning of surface structure J. Biomed. Mater. Res. Part B Appl. Biomater. 102 1580-94

[22] Qi L, Li N, Huang R, Song Q, Wang L, Zhang Q, Su R, Kong T, Tang M and Cheng G 2013 The effects of topographical patterns and sizes on neural stem cell behavior PLoS One 8 e59022

[23] Yang K, Jung H, Lee H R, Lee J S, Kim S R, Song K Y, Cheong E, Bang J, Im S G 
and Cho S W 2014 Multiscale, hierarchically patterned topography for directing human neural stem cells into functional neurons ACS Nano 8 7809-22

[24] Jaggy M, Zhang P, Greiner A M, Autenrieth T J, Nedashkivska V, Efremov A N, Blattner C, Bastmeyer M and Levkin P A 2015 Hierarchical Micro-Nano Surface Topography Promotes Long-Term Maintenance of Undifferentiated Mouse Embryonic Stem Cells Nano Lett. 15 7146-54

[25] Battiston K G, Cheung J W C, Jain D and Santerre J P 2014 Biomaterials in coculture systems: Towards optimizing tissue integration and cell signaling within scaffolds Biomaterials 35 4465-76

[26] Kaji H, Camci-Unal G, Langer R and Khademhosseini A 2011 Engineering systems for the generation of patterned co-cultures for controlling cell - cell interactions Biochim. Biophys. Acta 1810 239-50

[27] Hui E E and Bhatia S N 2007 Microscale Control of Cell Contact and Spacing via Three-Component Surface Patterning Langmuir 23 4103-7

[28] Yamazoe H, Ichikawa T, Hagihara Y and Iwasaki Y 2016 Generation of a patterned co-culture system composed of adherent cells and immobilized nonadherent cells Acta Biomater. 31 231-40

[29] Javaherian S, Li K and McGuigan A 2013 A simple and rapid method for generating patterned co-cultures with stable interfaces Biotechniques 55 21-5

[30] Stratakis E 2012 Nanomaterials by ultrafast laser processing of surfaces Sci Adv Mater 4 407-431

[31] Chichkov B N, Momma C, Nolte S, von Alvensleben F and Tünnermann A 1996 Femtosecond, picosecond and nanosecond laser ablation of solids Appl. Phys. A $\mathbf{6 3}$ $109-15$

[32] Tsibidis G D, Barberoglou M, Loukakos P A, Stratakis E and Fotakis C 2012 Dynamics of ripple formation on silicon surfaces by ultrashort laser pulses in subablation conditions Phys. Rev. B - Condens. Matter Mater. Phys. 86 1-14

[33] Tsibidis G D, Fotakis C and Stratakis E 2015 From ripples to spikes: A hydrodynamical mechanism to interpret femtosecond laser-induced self-assembled structures Phys. Rev. B - Condens. Matter Mater. Phys. 92 1-6

[34] Shen M Y, Crouch C H, Carey J E and Mazur E 2004 Femtosecond laser-induced formation of submicrometer spikes on silicon in water Appl. Phys. Lett. 85 5694-6

[35] Zhu S, Lu Y F, Hong M H and Chen X Y 2001 Laser ablation of solid substrates in water and ambient air J. Appl. Phys. 89 2400-3

[36] Tan J and Saltzman W M 2004 Biomaterials with hierarchically defined micro- and 
nanoscale structure Biomaterials 25 3593-601

[37] Huang Y, Zha G, Luo Q, Zhang J, Zhang F, Li X, Zhao S, Zhu W and Li X 2014 The construction of hierarchical structure on Ti substrate with superior osteogenic activity and intrinsic antibacterial capability Sci. Rep. 46172

[38] Kubo K, Tsukimura N, Iwasa F, Ueno T, Saruwatari L, Aita H, Chiou W A and Ogawa T 2009 Cellular behavior on TiO2 nanonodular structures in a micro-tonanoscale hierarchy model Biomaterials 30 5319-29 